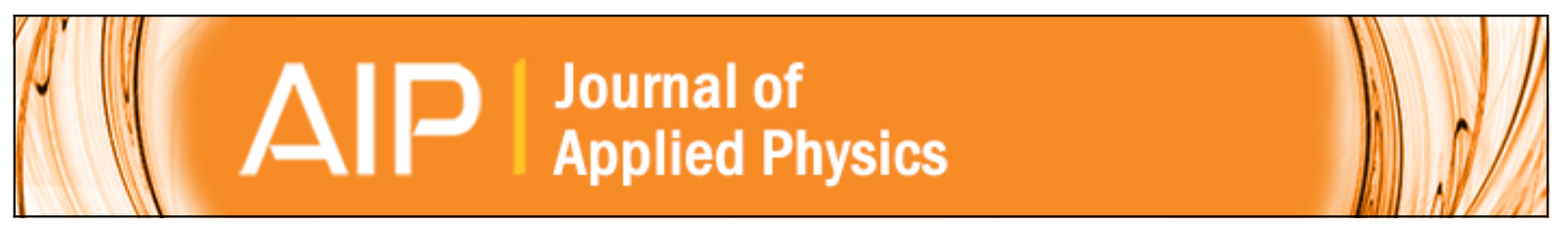

\title{
Unexpected short- and medium-range atomic structure of sputtered amorphous silicon upon thermal annealing
}

B. Haberl, S. N. Bogle, T. Li, I. McKerracher, S. Ruffell, P. Munroe, J. S. Williams, J. R. Abelson, and J. E. Bradby

Citation: Journal of Applied Physics 110, 096104 (2011); doi: 10.1063/1.3658628

View online: http://dx.doi.org/10.1063/1.3658628

View Table of Contents: http://scitation.aip.org/content/aip/journal/jap/110/9?ver=pdfcov

Published by the AIP Publishing

\section{Articles you may be interested in}

Impact of annealing induced structural relaxation on the electrical properties and the crystallization kinetics of amorphous GeTe films

Appl. Phys. Lett. 103, 071901 (2013); 10.1063/1.4818520

Metal-induced nanocrystalline structures in Ni-containing amorphous silicon thin films

J. Appl. Phys. 100, 094311 (2006); 10.1063/1.2362877

Hydrogen-induced modification of the medium-range structural order in amorphous silicon films

Appl. Phys. Lett. 87, 241915 (2005); 10.1063/1.2143124

Impact of the end of range damage from low energy Ge preamorphizing implants on the thermal stability of shallow boron profiles

J. Appl. Phys. 96, 4939 (2004); 10.1063/1.1776624

Evolution of structural order in germanium ion-implanted amorphous silicon layers

J. Appl. Phys. 92, 910 (2002); 10.1063/1.1486256

\section{AIP $\left.\right|_{\text {APL Photonics }}$}

APL Photonics is pleased to announce Benjamin Eggleton as its Editor-in-Chief

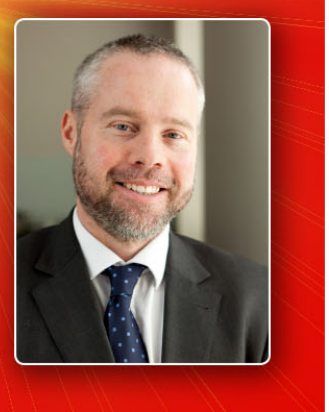




\title{
Unexpected short- and medium-range atomic structure of sputtered amorphous silicon upon thermal annealing
}

\author{
B. Haberl, ${ }^{1, a)}$ S. N. Bogle, ${ }^{2}$ T. Li, ${ }^{2}$ I. McKerracher, ${ }^{1}$ S. Ruffell, ${ }^{1}$ P. Munroe,${ }^{3}$ J. S. Williams, ${ }^{1}$ \\ J. R. Abelson, ${ }^{2}$ and J. E. Bradby ${ }^{1}$ \\ ${ }^{1}$ Department of Electronic Materials Engineering, Research School of Physics and Engineering, \\ The Australian National University, Canberra, ACT 0200, Australia \\ ${ }^{2}$ Department of Materials Science and Engineering, University of Illinois at Urbana-Champaign, \\ Urbana, Illinois 61801, USA \\ ${ }^{3}$ Electron Microscope Unit, University of New South Wales, Sydney, NSW 2052, Australia
}

(Received 2 August 2011; accepted 3 October 2011; published online 7 November 2011)

\begin{abstract}
We investigate the structure of magnetron-sputtered (MS) amorphous silicon (a-Si) prepared under standard deposition conditions and compare this to pure ion-implanted (II) a-Si. The structure of both films is characterized in their as-prepared and thermally annealed states. Significant differences are observed in short- and medium-range order following thermal annealing. Whereas II a-Si undergoes structural relaxation toward a continuous random network, MS a-Si exhibits little change. Cross-sectional transmission electron microscopy reveals the presence of nanopores in the MS film consistent with reduced mass-density. Therefore, the short- and medium-range order of annealed, MS a-Si is tentatively attributed to these pores. (C) 2011 American Institute of Physics. [doi:10.1063/1.3658628]
\end{abstract}

The structure of amorphous silicon (a-Si) has attracted wide interest over the last four decades, ${ }^{1-4}$ since a-Si is a model covalently bonded amorphous system. Previously, a-Si prepared by different methods and with different histories has been observed to exhibit different short-range order (SRO) as well as medium-range order (MRO) characteristics. For example, ion-implanted (II) a-Si displays a high degree of MRO, whereas pressure-induced (PI) a-Si exhibits a significantly reduced MRO, ${ }^{4}$ but increased mass density. ${ }^{5}$ This reflects the dramatically different formation methods of these two pure forms of a-Si.

Another crucial parameter for the structural properties of an a-Si film is its thermal history. For example, when subjected to ex-situ thermal annealing, II a-Si undergoes short-range ordering or "structural relaxation" toward an ideal continuous random network $(\mathrm{CRN})^{2,3}$ As suggested by Roorda et al., ${ }^{2,3}$ this can be attributed to defect annihilation. More recently, it has been observed by fluctuation electron microscopy $(\mathrm{FEM})^{6}$ that these structural changes upon annealing also occur on the MRO scale, resulting in the removal of inhomogeneities, such as density differences, and even paracrystallites, and thus yielding a more homogeneous $\mathrm{CRN}^{4,7}$ The relaxation toward a CRN is also reflected in mechanical measurements, whereby relaxed a-Si exhibits a phase transformation to crystalline end phases under low-load indentation, whereas unrelaxed a-Si deforms via plastic flow. ${ }^{8}$ Interestingly, this structural relaxation is not unique to II a-Si, as PI a-Si also undergoes structural relaxation toward a CRNlike network upon thermal annealing. ${ }^{4}$

The situation with deposited forms of a-Si is significantly more complex, since the exact deposition conditions often control the observed behavior. For example, impure a$\mathrm{Si}$, such as hydrogenated a-Si, does not phase transform

${ }^{a}$ Electronic mail: bianca.haberl@anu.edu.au. under indentation, even when "relaxed" by a thermal anneal. ${ }^{5}$ In contrast, films deposited under ultrahigh vacuum conditions behave like pure II a-Si, for example, in terms of epitaxial laser crystallization, ${ }^{9}$ and it might be expected that indentation behavior and SRO/MRO properties are similar to II a-Si. However, such deposition conditions are not routine. Therefore, the question arises as to whether a-Si films with low impurity content $(<1$ at. \%), but deposited under standard laboratory conditions have similar relaxation behavior and SRO/MRO properties.

Thus, the aim of this study is to investigate the structural properties (i.e., SRO and MRO) of magnetron-sputtered (MS) a-Si deposited under standard laboratory conditions in comparison with pure II a-Si as a function of thermal history. The structural properties are characterized using nanoindentation, Raman microspectroscopy, and FEM. In contrast to previous FEM studies on II a-Si, ${ }^{4,7}$ this current study is performed employing a scanning transmission electron microscope (STEM). In addition, impurity content, density, and microstructure of the MS a-Si are determined using secondary ion mass spectrometry (SIMS), Rutherford backscattering spectrometry (RBS), and cross-sectional transmission electron microscopy (XTEM).

A continuous 2- $\mu \mathrm{m}$-thick surface amorphous layer of II a-Si was created at liquid nitrogen temperature using the ANU 1.7 MV NEC tandem high energy ion-implanter using a range of implant energies. ${ }^{4}$ The MS material was deposited with an AJA ATC 2400-V system using a 3-inch Si target at a nominal temperature of $300^{\circ} \mathrm{C}$. An RF power of $300 \mathrm{~W}$ was applied, producing a nominal cathode potential of 130 $\mathrm{V}$. The sputtering was performed in $3.7 \mathrm{mT}$ of Ar flowing at $20 \mathrm{sccm}$, and the deposition rate was $\sim 2.7 \mathrm{~nm} / \mathrm{min}$. A film thickness of $\sim 500 \mathrm{~nm}$ was deposited onto Czochralski grown (Cz) $\mathrm{Si}(100)$. Ex-situ anneals were performed at $450^{\circ} \mathrm{C}$ for $30 \mathrm{~min}$ in $\mathrm{N}_{2}$ on half of each of the a-Si samples. These 

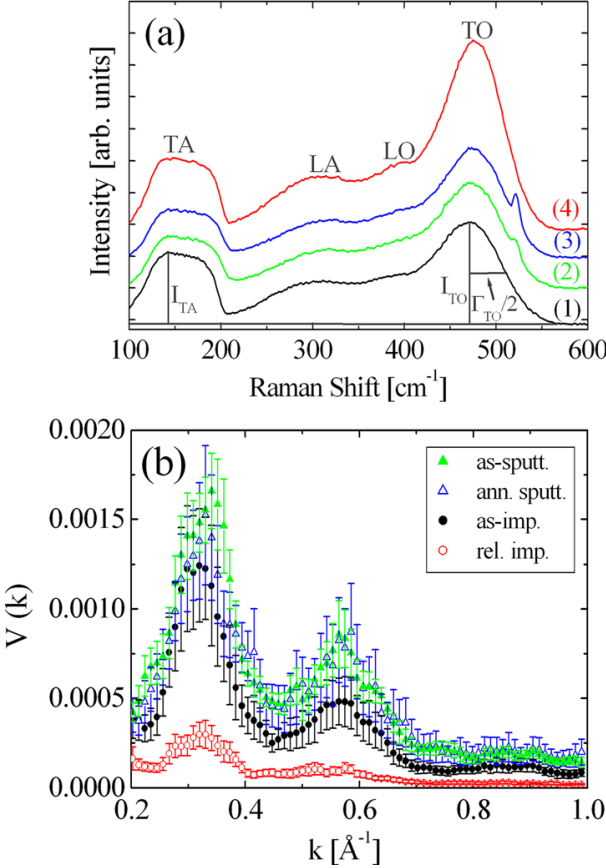

FIG. 1. (Color online) (a) Raman spectra of (1) as-implanted, (2) as-sputtered, (3) annealed sputtered, and (4) relaxed (annealed) implanted a-Si. The intensities of the TA-like and TO-like peaks as well as $\Gamma_{T O} / 2$ are indicated. (b) FEM $V(k)$ for as-prepared and annealed MS and II a-Si.

conditions are sufficient to fully relax II a-Si without recrystallizing the amorphous layer. 8,10

Nanoindentation was conducted on the MS a-Si films employing an Ultra-Micro-Indentation- System 2000 (UMIS). Standard conditions were chosen which are well known to result in a phase transformation to crystalline end phases in relaxed II a-Si. ${ }^{8}$ Selected indents were prepared for XTEM using an FEI xT Nova NanoLab 200 dual-beam focused ion beam system (FIB). ${ }^{4}$ A Philips CM 300, operating at an accelerating voltage of $300 \mathrm{kV}$, was used for conventional TEM imaging.

Raman microspectroscopy was conducted with a Renishaw 2000 Raman imaging microscope using the $632.8 \mathrm{~nm}$ excitation line of a helium-neon laser. ${ }^{4}$ For each sample, five areas were measured, and the results are the averages from these. ${ }^{5}$

Plan-view specimens of as-prepared and annealed II and MS a-Si were prepared for FEM. ${ }^{4,5}$ A JEOL 2200FS STEM was used for the FEM study employing a probe of $1.6 \mathrm{~nm}$. Nanodiffraction patterns were collected in a 15 x 15 square grid of at least $10 \mathrm{~nm}$ spacing and five such areas were scanned per sample. The intensity variance, $V(k)$, was computed for each area. ${ }^{11}$ The thickness was monitored by the normalized bright-field contrast and estimated as $\sim 50 \mathrm{~nm}$ and the individual areas were corrected for roughness. ${ }^{12}$ The remaining nanodiffraction patterns $(>500)$ were sufficient for adequate statistics, where the FEM data and peak ratios are the averages of the five or more areas measured.

The Raman spectra for (1) as-implanted, (2) as-sputtered, (3) annealed sputtered, and (4) relaxed implanted a-Si are shown in Fig. 1(a). The typical Raman features of a-Si, the broad transverse acoustic (TA)-like, longitudinal acoustic (LA)-like, longitudinal optic (LO)-like, and transverse optic (TO)-like peaks, are marked in Fig. 1(a). From these spectra, the bond-angle distortion $\Delta \theta$ can be determined ${ }^{1,2,13,14}$ from the TO peak half-width, $\Gamma_{T O} / 2$. In addition, the order beyond the SRO scale at a length of $\sim 4-6 \AA$ can be accessed from the intensity ratio of the TA to TO peaks. ${ }^{1,14,15}$ These characteristics are presented in Table I. In the case of II a-Si, $\Gamma_{T O} / 2$ decreases significantly upon annealing, as is typical. ${ }^{1,2} \mathrm{~A}$ reduction of $I_{T A} / I_{T O}$ by $\sim 60 \%$ is, consistent with previous studies, ${ }^{1}$ also observed upon annealing, indicating ordering on the length-scale of $\sim 4-6 \AA$. In the case of the MS a-Si, however, $\Gamma_{T O} / 2$ only decreases by $\sim 3 \%$ upon annealing and $I_{T A} / I_{T O}$ by $\sim 10 \%$, although both MS networks exhibit higher structural order beyond the SRO scale than the as-implanted case.

FEM data for the different forms of a-Si are shown in Fig. 1(b). This data suggest that only the as-sputtered MS film may exhibit some paracrystallinity, while the other cases do not. ${ }^{4,5,16}$ It is clear that annealing (relaxation) has a dramatic effect on peak magnitudes in the II a-Si case, but the MS a-Si exhibits little change. In the case of II a-Si, decrease of MRO upon relaxation is consistent with previous studies. ${ }^{4,7}$ For the as-prepared MS and II cases, the magnitudes of the first peak are very similar, but significant differences are observed for the magnitudes of the second peak, yielding significantly different peak magnitude ratios (Table I, last row). The ratio of $\sim 0.5$ for both the MS cases is consistent with previous STEM-FEM studies on deposited $\mathrm{a}-\mathrm{Si}^{11}$ and was interpreted as evidence of paracrystallinity. In contrast, the ratio of $\sim 0.35$ for both the II cases is significantly lower. The reasons for this very low ratio are not entirely clear yet.

Bright-field XTEM images of residual indents made in as-prepared and annealed MS a-Si are shown in Fig. 2. The film was "diffraction amorphous," and an selected area diffraction pattern (SADP) taken from beneath the residual indent from annealed MS a-Si is shown as inset in Fig. 2(b). No evidence of phase transformation is observed in the MS aSi films, which deform plastically. This is confirmed by Raman microspectroscopy (not shown). Significant slip and extended defect formation in the underlying crystalline $\mathrm{Si}$ is observed below the indent in annealed MS a-Si, which may indicate that this annealed film is somewhat harder.

Two regions, indicated in Fig. 2(a), are shown magnified with enhanced contrast in Fig. 3. The pristine layer shown in Fig. 3(a) clearly reveals the presence of vertical nanopores

TABLE I. Structural characteristics of the different forms of a-Si expressed in $\Gamma_{T O} / 2, \Delta \theta$ (after Beeman et al. (Ref. 13)), the ratio $I_{T A} / I_{T O}$ (all from Raman data), and the ratio of the magnitudes of peak two to one from the FEM data.

\begin{tabular}{lcccc}
\hline \hline Form of a-Si & As-implanted & Relaxed implanted & As-sputtered & Annealed sputtered \\
\hline$\Gamma_{T O} / 2\left[\mathrm{~cm}^{-1}\right]$ & $39.8 \pm 0.4$ & $34.7 \pm 0.3$ & $39.0 \pm 0.5$ & $37.8 \pm 0.3$ \\
$\Delta \theta[\mathrm{deg}]$ & $10.8 \pm 0.1$ & $9.1 \pm 0.1$ & $10.5 \pm 0.2$ & $10.1 \pm 0.1$ \\
$I_{T A} / I_{T O}$ & $0.636 \pm 0.019$ & $0.395 \pm 0.006$ & $0.501 \pm 0.003$ & $0.454 \pm 0.003$ \\
FEM peak ratio & $0.38 \pm 0.03$ & $0.32 \pm 0.03$ & $0.48 \pm 0.06$ & $0.55 \pm 0.08$ \\
\hline \hline
\end{tabular}



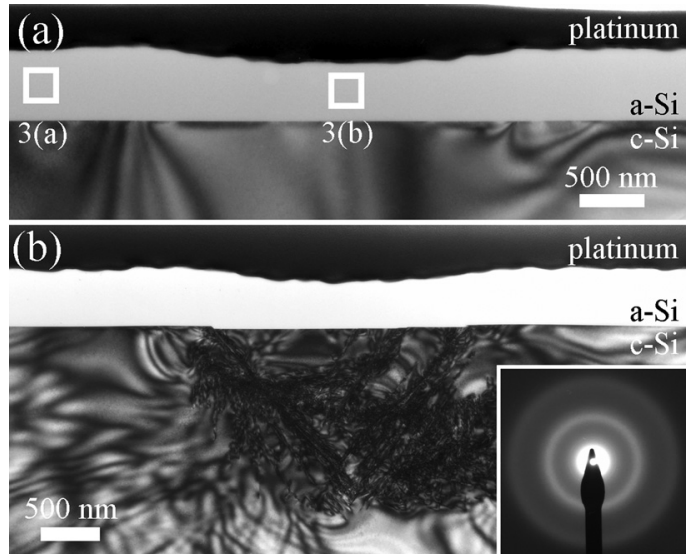

FIG. 2. Bright-field micrographs of residual indents made in (a) as-prepared and (b) annealed MS a-Si with an SADP taken from beneath the residual indent shown as an inset in the latter case.

(arrowed for clarity). In contrast, no nanopores are visible beneath the residual indent [Fig. 3(b)], behavior which is accompanied by a significant decrease $(\sim 10 \%)$ in normalized bright-field image intensity of the film. As the film thickness of this cross-section prepared by FIB is uniform, this decrease may be attributed to densification of the film, suggesting a significant density deficit in the as-sputtered film.

From SIMS data, ${ }^{17}$ the MS a-Si film contains $\sim 2.7$ at. $\%$ of $\mathrm{H}$ and $\mathrm{O}$ in total prior to annealing and 2.1 at. \% after annealing. In addition to these $\mathrm{O}$ and $\mathrm{H}$ impurities, sputterdeposition with argon is well known to result in the incorporation of Ar into the film, ${ }^{18}$ which is measured to be $\sim 2.0$ at. $\%$ by RBS performed on the ANU 1.7 MV Tandem accelerator using $2 \mathrm{MeV} \mathrm{He}^{+}$ions for analysis. Further RBS analysis in combination with XTEM measured a $\sim 5 \%$ lower mass density for the as-sputtered MS case compared to II a-Si (which is known to be void-free). ${ }^{19}$

Structural characterization of as-prepared II and MS a$\mathrm{Si}$, prepared under standard laboratory deposition conditions, and their annealed counterparts, reveals intriguing differences and similarities. Consider first the nature of the order in the as-prepared states. The $\Delta \theta$ of both as-prepared forms and the degree of MRO appear similar. The MS a-Si, however, is more ordered on the 4-6 $\AA$ scale than II a-Si, and also the $n a$ ture of the order on the MRO scale, as indicated by different FEM peak ratios, is different. As expected, together with the significant $\Delta \theta$ and degree of SRO observed by Raman, the FEM data indicates that both as-prepared networks are, at best, imperfect CRNs. Presumably, this is a result of both an inhomogeneous network on the MRO scale, ${ }^{4}$ as well as the presence of a large number of structural defects on the SRO

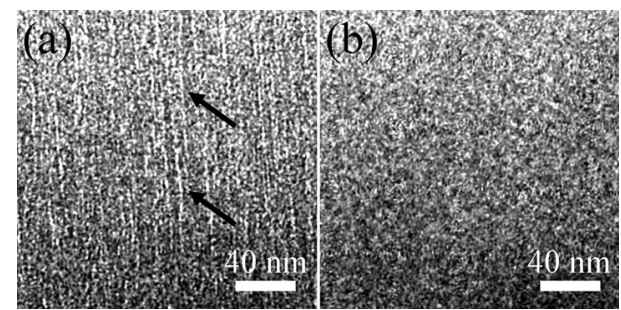

FIG. 3. Magnified regions from (a) the pristine as-prepared MS film and from (b) beneath the residual indent, as indicated in Fig. 2. scale. ${ }^{2,3}$ We speculate that, for the MS film, the observation of nanopores from TEM images and its lower density may be the reason for a different FEM peak ratio. It is noteworthy that modeling of porous a-Si has suggested that the material between the pores is considerably more ordered than in a comparable non-porous (amorphous) material, since the dangling bonds concentrate on the internal surfaces of the pores. ${ }^{20}$ This observation might also help to explain the higher SRO present in the MS a-Si compared to the II case.

Now consider the influence of ex-situ annealing on the respective networks. Whereas II a-Si undergoes structural relaxation toward a CRN, with large reduction in $\Delta \theta$ and MRO, MS a-Si undergoes only some small (but incomplete) short-range ordering. This lack of structural relaxation is also highlighted by the lack of phase transformation upon indentation testing and the remnant high degree of MRO from the FEM data. We suggest that it is not the impurity content of MS a-Si that governs this behavior, since II a-Si enriched with similar impurity contents is still observed to phase transform. ${ }^{21,22}$ Indeed, some ordering is observed by Raman microspectroscopy, which may be consistent with small-scale structural relaxation of the material between the pores. However, microscopic nanopores on the MRO scale may inhibit the atomic rearrangement necessary for complete relaxation (and homogenization of the network) and thus prevent convergence to a CRN.

In summary, comparison of the SRO and MRO structure of MS a-Si deposited under standard laboratory conditions with that of II a-Si has uncovered some interesting differences. Whereas both forms in their as-prepared state are similarly disordered on the SRO-scale and both exhibit a relatively high MRO, upon annealing, II a-Si undergoes structural relaxation toward a CRN, whereas the MS a-Si is little changed. We interpret that the porosity present in the as-prepared film inhibits further structural change on thermal annealing.

${ }^{1}$ J. Fortner and J. Lannin, Phys. Rev. B 37, 10154 (1988).

${ }^{2}$ S. Roorda et al., Phys. Rev. B 44, 3702 (1991).

${ }^{3}$ K. Laaziri et al., Phys. Rev. B 60, 13520 (1999).

${ }^{4}$ B. Haberl et al., Phys. Rev. B 79, 155209 (2009).

${ }^{5}$ B. Haberl, "Structural characterization of amorphous silicon," Ph.D. thesis, Australian National University, 2010.

${ }^{6}$ M. M. J. Treacy et al., Rep. Prog. Phys. 68, 2899 (2005).

${ }^{7}$ J.-Y. Cheng et al., J. Mater. Res. 16, 3030 (2001).

${ }^{8}$ B. Haberl et al., J. Appl. Phys. 100, 013520 (2006).

${ }^{9}$ J. C. Bean et al., J. Appl. Phys. 50, 881 (1980).

${ }^{10}$ G. L. Olson and J. A. Roth, Mater. Sci. Rep. 3, 1 (1988).

${ }^{11}$ S. N. Bogle et al., Ultramicroscopy 110, 1273 (2010).

${ }^{12}$ S. N. Bogle, "Quantifying nanoscale order in amorphous materials via fluctuation electron microscopy," Ph.D. thesis, University of Illinois at Urbana-Champaign, 2009.

${ }^{13}$ D. Beeman, R. Tsu, and M. F. Thorpe, Phys. Rev. B 32, 874 (1985).

${ }^{14}$ M. Marinov and N. Zotov, Phys. Rev. B 55, 2938 (1997).

${ }^{15}$ A. P. Sokolov et al., J. Phys. Condens. Matter 3, 9887 (1991).

${ }^{16} \mathrm{P}$. M. Voyles, M. M. J. Treacy, and J. M. Gibson, in New Methods, Mechanisms, and Models of Vapor Deposition, Materials Research Society Symposium Proceedings Vol. 616, edited by H. N. G. Wadley, G. H. Gilmer, and W. G. Barker (MRS, Warrendale, PA, 2000), pp. 47-52.

${ }^{17}$ See http://www.eaglabs.com/ for information about Evans Analytical Group LLC.

${ }^{18}$ M. Ohring, Materials Science of Thin Films, 2nd ed. (Academic, San Diego, 2002).

${ }^{19}$ D. L. Williamson et al., Appl. Phys. Lett. 67, 226 (1995).

${ }^{20}$ G. Opletal et al., J. Chem. Phys. 126, 214705 (2007).

${ }^{21}$ S. Ruffell et al., J. Appl. Phys. 105, (2009).

${ }^{22}$ S. Ruffell et al., J. Appl. Phys. 106, 123511 (2009). 